\title{
Optimizing NK-92 Serial Killers: Gamma Irradiation, CD95/FasLigation, And NK Or LAK Attack Limit Cytotoxic Efficacy
}

\section{Lydia T Navarrete-Galvan}

University of Nevada https://orcid.org/0000-0003-1736-8867

\section{Michael Guglielmo}

Fred Hutchinson Cancer Research Center

Judith Cruz Amaya

University of Nevada

Julie Smith-Gagen

University of Nevada

Vincent C. Lombardi

University of Nevada

Rebecca Merica

St. Olaf College, Northfield

Dorothy Hudig ( $\nabla$ dhudig@med.unr.edu )

University of Nevada https://orcid.org/0000-0001-5952-208X

\section{Research Article}

Keywords: NK-92, NK, radiation, Fas/CD95, serial killing, lymphokine activated killer, limitations, adoptive cell transfer, therapeutic efficacy

Posted Date: December 28th, 2021

DOI: https://doi.org/10.21203/rs.3.rs-1193866/v1

License: (c) (1) This work is licensed under a Creative Commons Attribution 4.0 International License. Read Full License 


\section{Optimizing NK-92 Serial Killers: Gamma Irradiation, CD95/Fas- Ligation, and NK or LAK Attack Limit Cytotoxic Efficacy}

Lydia Navarrete-Galvan', Michael Guglielmo², Judith Cruz Amaya ${ }^{1}$, Julie Smith-Gagen ${ }^{3}$, Vincent C. Lombardi ${ }^{1}$, Rebecca Merica ${ }^{4}$, Dorothy Hudig ${ }^{*}$

${ }^{1}$ University of Nevada, Reno School of Medicine, Reno, NV, 89557, USA.

${ }^{2}$ Fred Hutchinson Cancer Research Center, Seattle, WA, 98109, USA.

${ }^{3}$ University of Nevada, Reno School of Community Health Sciences, Reno, NV 89557, USA.

${ }^{4}$ Biology Department, St. Olaf College, Northfield, MN, 55057, USA.

*Correspondence: dhudig@med.unr.edu 


\section{Abstract}

2 Background: The NK cell line NK-92 and its genetically modified variants are receiving attention as

3 immunotherapies to treat a range of malignancies. However, since NK-92 cells are themselves tumors,

4 they require irradiation prior to transfer and are potentially susceptible to attack by patients' immune

5 systems. Here, we investigated NK-92 cell-mediated serial killing for the effects of gamma-irradiation

6 and ligation of the death receptor Fas (CD95), and NK-92 cell susceptibility to attack by activated

7 primary blood NK cells.

8 Methods: To evaluate serial killing, we used ${ }^{51}$ Cr-release assays with low NK-92 effector cell to target

9 Raji, Daudi or K562 tumor cell (E:T) ratios to determine killing frequencies at 2-, 4-, 6-, and 8-hours.

10 Results: NK-92 cells were able to kill up to 14 Raji cells per NK-92 cell in eight hours. NK-92 cells

11 retained high cytotoxic activity immediately after irradiation with 10 Gy but the cells surviving irradiation

12 lost $>50 \%$ activity one day after irradiation. Despite high expression of CD95, NK-92 cells maintained

13 their viability following overnight Fas/CD95-ligation but lost some cytotoxic activity. However, one day

14 after irradiation, NK-92 cells were more susceptible to Fas ligation, resulting in decreased cytotoxic

15 activity of the cells surviving irradiation. Irradiated NK-92 cells were also susceptible to killing by both

16 unstimulated and IL-2 activated primary NK cells (LAK). In contrast, non-irradiated NK-92 cells were

17 more resistant to attack by NK and LAK cells.

18 Conclusions: Irradiation is deleterious to both the survival and cytotoxicity mediated by NK-92 cells and

19 renders the NK-92 cells susceptible to Fas-initiated death and death initiated by primary blood NK cells.

20 Therefore, replacement of irradiation as an antiproliferative pretreatment and genetic deletion of Fas

21 and/or NK activation ligands from adoptively transferred cell lines are indicated as new approaches to

22 increase therapeutic efficacy.

23 Keywords: NK-92, NK, radiation, Fas/CD95, serial killing, lymphokine activated killer, 24 limitations, adoptive cell transfer, therapeutic efficacy

25 Abbreviations: ADCC, antibody dependent cellular cytotoxicity; CAR, chimeric antigen receptor; E:T,

26 effector to target ratio; IL-2, interleukin-2; KF, killing frequency; LAK, lymphokine-activated killer; 
$27 \mathrm{LU}_{50}$, lytic units as the number of cells required to kill $50 \%$ of the 'target' cells; mAb, monoclonal

28 antibody; NK, natural killer, including bNK as primary blood NK cell; PBMC, peripheral blood

29 mononuclear cells.

\section{Background}

31 Natural killer (NK) cells are appealing cells for immunotherapy because they are very potent effector

32 lymphocytes of the innate immune system that can attack and kill many different tumor target cells

33 without prior sensitization (1-3). Studies show that some cytotoxic immune cells, including T- and NK

34 cells, are capable of killing multiple target cells, sequentially, in a process termed serial killing $(4,5)$.

Moreover, serial killer cells are often faster at delivering lytic hits and inducing target cell death than nonserial killer NK cells (6). Unfortunately for adoptive immunotherapies, many NK cells kill only once,

37 with less than $30 \%$ capable of serial killing (7-9). While the number of target cells killed per effector varies by study, it is consistently noted that a minority of NK cells is responsible for the majority of killing events. Furthermore, there are challenges to obtaining sufficient numbers of functionally active NK cells from a patient's blood because, despite being widespread throughout the body, NK cells

41 represent just $2-18 \%$ of lymphocytes in human peripheral blood and it is challenging to obtain sufficient 42 numbers of NK cells needed to overwhelm the number of tumor cells $(10,11)$.

43 To readily obtain the large numbers of NK cells needed, immortalized cytotoxic cell lines have been 44 established from patients with NK-cell cancers; however, little is known about their serial killing 45 capacities. NK-92 are one of over eight available NK cell lines (Additional File 1: Table S1) and have 46 reproducible cytotoxicity to a variety of tumor types $(10,12,13)$, even under hypoxic conditions (14).

$47 \quad$ NK-92 cells can also be genetically manipulated to express receptors that recognize specific tumor antigens and that augment therapeutic monoclonal antibodies through antibody-dependent cellular cytotoxicity (ADCC). These NK-92 cells and CAR-NK-92 variants are immediately available and more affordable than current CAR-T-cell therapy (15). In fact, haNK (NK-92) cells, engineered to express the high affinity CD16A allele (in order to recognize tumor cell-bound monoclonal antibodies), were tested in 
combination with anti-PD-L1 antibody, avelumab, and have now been further modified to also express a

PD-L1-specific chimeric antigen receptor $(16,17)$. NK-92 cells have been infused into patients with advanced cancers, resulting in clinical benefits with limited side effects. Additionally, NK-92 cells are being tested in several clinical trials in four different countries and for patients with a range of malignancies, including leukemia, glioblastoma, and melanoma (18-21). It is important to assess what can happen to these cells in vivo, following transfer, a challenging issue that has been addressed so far only by monitoring cells circulating in the patients' blood (19). In this report, we assessed in vitro, potential hazards to NK-92 cell serial killing that could occur in vivo after adoptive transfer, including losses of cytotoxic serial capacity following irradiation, ligation of NK-92 cell Fas by cells residing in the tumor, as well as vulnerability of the NK-92 cells to attack by blood primary NK cells.

As far as the authors are aware, we are the first to observe killing frequencies (5) (KF) $>1$ by NK-92 cells using standard release assays (presented at American Association of Immunologists annual meeting, 2021). We monitored this serial killing to predict potential losses of activity to the cells during the time that the cells remain viable after adoptive transfer. Our data demonstrate several potential complications that would result in losses to therapeutic efficacy in vivo when NK-92 cells become impaired following irradiation. It was previously reported that when NK-92 cells were irradiated with 10 Gy, NK-92 cell proliferation was prevented and cytolytic activity was substantially conserved within the live cells remaining one day following irradiation (22). We found, however, that NK-92 cell serial killing significantly decreased one day after irradiation. Irradiation also increased NK-92 cell susceptibility to Fas-ligation as well as to attack by lymphokine-activatable primary blood NK cells.

\section{Methods}

Cell Lines and Culture. All cell lines were regularly tested negative for mycoplasma using the MycoAlert ${ }^{\mathrm{TM}}$ mycoplasma detection kit (Lonza, Walkersville MD). NK-92 cells (ATCC CRL-2407) were cultured in alpha Minimum Essential Media with L-glutamine and sodium pyruvate, no 
ribonucleosides or deoxyribonucleosides (Gibco, Waltham MA), with $0.2 \mathrm{mM}$ inositol, $0.2 \mathrm{mM} 2$ mercaptoethanol, and $0.02 \mathrm{mM}$ folic acid, $12.5 \%$ horse serum (Gibco), 12.5\% fetal bovine serum (FBS) (Atlanta Biologicals, Flowery Branch GA), and 1000 U/ml Tecin, Teceleukin recombinant interleukin 2 (IL-2) (Roche, Basel Switzerland) at 5\% $\mathrm{CO}_{2}$ and $37^{\circ} \mathrm{C}$. $\mathbf{K 5 6 2}$ cells (ATCC CCL-243) were cultured in Dulbecco's modified Eagle Medium (DMEM) with $4.5 \mathrm{~g} / \mathrm{L}$ glucose, L-glutamine, and sodium pyruvate (Corning Life Sciences) and with 10\% fetal bovine serum (FBS) and 1\% penicillin-streptomycin (penstrep) solution (MilliporeSigma, Burlington MA) at $5 \% \mathrm{CO}_{2}$ and $37{ }^{\circ} \mathrm{C}$. Raji (ATCC CCL-86), Daudi (ATCC CCL-213), and Jurkat (ATCC TIB-152) cells were cultured in Roswell Park Memorial Institute (RPMI) media with L-glutamine (Corning Life Sciences, Tewksbury MA), 10\% FBS and 1\% pen-strep at 5\% $\mathrm{CO}_{2}$ and $37^{\circ} \mathrm{C}$. PBMCs (STEMCELL Technologies, Vancouver, Canada) were either resting, unstimulated after culture in DMEM with $4.5 \mathrm{~g} / \mathrm{L}$ glucose, L-glutamine, and sodium pyruvate and with $10 \%$ FBS and $1 \%$ Pen-Strep at $37{ }^{\circ} \mathrm{C}$ and $5 \% \mathrm{CO}_{2}$ overnight or LAK activated, cultured in DMEM with $4.5 \mathrm{~g} / \mathrm{L}$ glucose, L-glutamine, and sodium pyruvate and with $10 \% \mathrm{FBS}$ and $1 \%$ pen-strep at $37{ }^{\circ} \mathrm{C}$ and $5 \%$ $\mathrm{CO}_{2}$ and supplemented with $1000 \mathrm{U} / \mathrm{ml} \mathrm{IL}-2$ for three days prior to assay.

Cell Irradiation. NK-92 cells were gamma irradiated in $15 \mathrm{ml}$ of culture media, using a cesium ${ }^{137}$ source (JL Shepherd Model I-30). A dose range of 2.5 - 20 Gy was tested. After radiation, cells were cultured for the times indicated ( $0-48$ hours) in $1000 \mathrm{U} / \mathrm{ml} \mathrm{IL-2.} \mathrm{Control} \mathrm{cells} \mathrm{were} \mathrm{processed} \mathrm{in} \mathrm{parallel} \mathrm{without}$ applying irradiation.

Fas-receptor Ligation. For cytotoxicity assays, NK-92 cells were brought to $2.5 \times 10^{5}$ cells $/ \mathrm{ml}$ and cultured overnight with either $1 \mathrm{ug} / \mathrm{ml}$ LEAF purified anti-human CD95 clone EOS9.1 or LEAF purified mouse IgM clone MM-30 as an isotype control. For flow cytometric assays of potential cell death induced after ligation of cellular Fas/CD95, NK-92 or Jurkat cells were cultured overnight at $2.0 \times 10^{6}$ cells/ml with $1 \mathrm{ug} / \mathrm{ml}$ LEAF purified anti-human CD95 or LEAF purified mouse IgM isotype control. When combined with irradiation, cells were first irradiated and then mAbs were added either immediately after irradiation or one day after irradiation, as indicated in the results. Control cells were processed in 
parallel without addition of antibodies. The mAbs were from BioLegend (San Diego, CA).

102 Cytotoxicity Assays. Target cells were labeled with $\mathrm{Na}^{51} \mathrm{CrO}_{4}$ (Perkin Elmer, Waltham, MA) (23).

103 Effector NK-92 cell counts were determined using a hemacytometer with routine samples of $>600$ cells

104 that excluded Trypan blue (MilliporeSigma). NK-92 effector cells were diluted 2-fold in quadruplicate in

105 V-bottom plates (Costar 3894, 96 well) in $0.1 \mathrm{ml}$ to create six to eight effector to target cell (E:T) ratios.

106 NK cell effectors within PBMCs were diluted similarly. Radiolabeled 'target' cells $\left(1 \times 10^{5} / \mathrm{ml}\right.$ in $\left.0.1 \mathrm{ml}\right)$

107 were added to each well to induce cytotoxicity. Plates were centrifuged (Sorvall RC 6+) at $1000 \mathrm{rpm}$ for

108 three minutes to bring the effector and target cells together and incubated at $5 \% \mathrm{CO}_{2}$ and $37{ }^{\circ} \mathrm{C}$ for eight

109 hours (unless otherwise noted). After incubation, plates were centrifuged at $1200 \mathrm{rpm}$ for 10 minutes and

$1100.1 \mathrm{ml}$ of cell-free supernatant was removed for analysis in a Perkin-Elmer Wizard gamma counter.

111 Spontaneous release was calculated using the average leak rate of target cells without effectors and the

112 maximum release was the radioactivity released by target cells lysed with $1 \%$ SDS. The calculated $\%$

113 specific release is a measure of target cell killing, as targets release internalized ${ }^{51} \mathrm{Cr}$ into the sampled

114 supernatant when they die. Percent specific release was calculated using the following formula:

$115 \%$ Specific Release $=[($ Experimental counts - Spontaneous Release $) /($ Max - Spontaneous Release $)] \mathrm{x}$

116 100. The data illustrated are representative of a minimum of three replicate experiments.

117 Flow Cytometry Analysis. For determining the presence of Fas-receptor CD95 on cells, the AF647

118 anti-CD95 clone DX2 was used with Zombie Aqua to eliminate dead cells from consideration. Cells

119 were taken immediately from culture, washed once with PBS, and stained with a 1:100 dilution of

120 Zombie Aqua for $30 \mathrm{~min}$ at room temperature (RT), protected from light. Then cells were quenched with

121 FACS buffer with $1 \%$ FCS and brought to $5 \times 10^{6}$ cells $/ \mathrm{ml}$, aliquoted into flow tubes, stained with 10

$122 \mathrm{ug} / \mathrm{ml}$ AF647 anti-CD95 clone DX2 for $30 \mathrm{~min}$ at RT, protected from light and washed twice. For

123 analysis of cell death following irradiation and/or anti-Fas ligation, the following fluorescent probes

124 were used: FITC annexin V (BioLegend, San Diego CA) and 7-aminoactinomycin D (7-AAD) 
(MilliporeSigma). Irradiated or anti-Fas treated, and control cells were washed with annexin V binding buffer and stained with $4 \mathrm{ug} / \mathrm{ml}$ 7-AAD and $4.5 \mathrm{ug} / \mathrm{mL}$ FITC annexin $\mathrm{V}$ for $20 \mathrm{~min}$ at RT, protected from light, then washed twice and brought up with annexin $\mathrm{V}$ buffer containing $20 \mathrm{ug} / \mathrm{ml}$ actinomycin D (AD) (MilliporeSigma), and fixed with $0.5 \%$ formaldehyde. For determining NK counts within PBMCs, TruCOUNT ${ }^{\mathrm{TM}}$ Beads (BD Biosciences) and the following fluorescent antibody panel was used: PacBlue anti-CD45 clone HI30, BV711 anti-CD56 clone HCD56, BV711 anti-CD16A clone 3G8, FITC anti-CD3 clone OKT3, AF647 anti-CD244 (24) clone C1.7, together with 7-AAD to identify necrotic cells, without washing the cells (in order to prevent cell loss). Gating sequence available in Additional File 2: Figure S1. Resting or LAK activated PBMCs (as previously described) were taken from culture, spun down, and resuspended in FACS buffer, then aliquoted into flow tubes. Cells were stained for $30 \mathrm{~min}$ at RT, protected from light. After staining $20 \mathrm{ug} / \mathrm{ml} \mathrm{AD}$ was added and the cells fixed by addition of formaldehyde (MilliporeSigma, Boston MA) to a final concentration of $0.5 \%$. All mAbs were from BioLegend, San Diego CA, and titrated for the concentrations suitable for no-wash conditions. The samples were analyzed within one day, using a BD Biosciences Special Order Research Product LSR II analytical flow cytometer with a high throughput sampler (HTS) unit. Cytometric data were analyzed with FlowJo software (BD Biosciences) to determine cell counts, \%positive cells, median fluorescence intensity (MFI), and statistical comparisons between samples. The data illustrated are representative of a minimum of two replicate experiments.

Statistical Analyses. Cytotoxicity assay data were calculated and graphed with Microsoft Excel and evaluated using SPSS Statistics (IBM, version 28, Armonk, NY) using linear regression analysis or difference-in-difference comparisons. LU 50 's (the number of effector cells needed to cause $50 \%$ lysis) were calculated by linear equations of cytotoxicity $\left(y=\%\right.$ specific ${ }^{51} \mathrm{Cr}$ release, $\mathrm{x}=\log _{10}$ of the E:T cell ratios) to determine the number of cells needed to kill $50 \%$ of the 'target' cells. Then the lytic activity was expressed as $\mathrm{LU}_{50} / 1.0 \times 10^{6}$ effector cells. FlowJo (BD Biosciences) “compare population” tool was used to calculate Overton subtraction (25) and chi-squared statistics to analyze flow cytometric populations. 


\section{Results}

\section{NK-92 cells serially kill multiple cancer cell lines}

152 Only some tumor cells are suitable targets for serial killing because NK cells sometimes lose their 153 receptors as they kill. The commonly used NK target cell, K562, was compared with two B-cell tumor 154 lines, Daudi and Raji, using killer frequencies (KFs) to measure the average number of tumor 'target' 155 cells killed per single NK-92 cell over time. The assays were stopped at 2-, 4-, 6-, and 8-hour time points 156 for measurement of cytotoxic activity. Raji cells were killed better than K562 and Daudi at all time157 points. Looking specifically at the 8-hour data and at an E:T of 1:16, Rajis have 57\% specific release (KF $158=9$ dead per single NK-92 cell, calculated as $56 \%$ of targets killed divided by $6.25 \%$, the frequency of 159 effectors available at a 1:16 ratio) compared to 53\% $(\mathrm{KF}=8.5)$ and just $17 \%(\mathrm{KF}=2.7)$ for Daudi and 160 K562 targets, respectively (Figure 1A-C). Additionally, Raji and Daudi tumor cells continued to be 161 serially killed after 6 hours unlike K562 cells, where serial killing stopped after 6 hours (Figure 1B and

162 C). For 8-hour assays, at an E:T of 1:16, NK-92 cells had an average KF of 6.7 Raji per effector in 11 163 experiments, with a KF range of 3.0 - 9.5. NK-92 KFs increased as target concentrations increased, 164 reaching a record of 14 Raji per NK-92 at an E:T of 1:32. The data in Figure 1 represent the serial killing 165 to each target that was observed within one experiment. Similar activities were observed within multiple 166 experiments (Raji: $n=11$ experiments, Daudi: $n=3, K 562: n=4)$.

167 Killing frequency (KF) describes the average number of targets killed per effector cell and assumes that 168 every effector cell kills (5). A single round of killing for each effector cell concentration is indicated by 169 the corresponding, colored dashed lines in Figure 1. It should be noted that in practice with NK-92 cells there is heterogeneity in killing. We observed variable externalization of CD107A associated with

171 cytotoxic granule release (26) (Navarrete-Galvan et al., unpublished results) and variability in killing was 172 also observed previously by time-lapse cinematography $(27,28)$. Therefore, it is likely that an individual 173 NK-92 cell can kill more targets than are reflected by the KF values. 
175 The cytotoxic capacity of NK-92 cells was measured following irradiation, either immediately or

176 following overnight culture, to detect activity of those cells that temporarily resist the effects of lethal

177 irradiation. We used 2.5 to $20 \mathrm{~Gy} ; 10 \mathrm{~Gy}$ is the FDA standard for adoptive transfer. Figure $2 \mathrm{~A}$ shows that

178 immediately after 20 Gy irradiation, NK-92 cells retained full killing capacity. However, following

179 overnight culture, the NK-92 effectors that were still viable showed a dose response of decreasing

180 cytotoxic functionality as radiation dosage increased. The effects on serial killing were pronounced. At

181 an E:T of 1:16, 35.0\% of targets were killed by non-irradiated NK-92 cells (KF 5.6) compared to 9.1\%

182 killing by 10 Gy irradiated NK-92 effectors (KF 1.5 ), a loss of more than $2 / 3$ of cytotoxic activity per cell

183 (Figure 2B, p-value $<0.001$ ). This dose-response radiation effect on serial killing was also observed with K562 targets (not illustrated). Thus, the serial killing by the NK-92 cells was greatly decreased by FDA-approved irradiation. It should be noted that at lower doses of irradiation than the $10 \mathrm{~Gy}$ in current clinical practice, serial killing was still significantly impacted; 2.5 Gy irradiated NK-92 cells had a KF of $<0.001)$.

Loss of cells due to radiation-induced death and loss of cytotoxic functionality act synergistically to limit the overall efficacy of irradiated NK-92 cells. When loss of serial killing is considered in combination with lower viable cell recovery, $10 \%$ of the potential NK-92 serial killing efficacy was retained one day

192 after 10 Gy irradiation (Table 1). Using lytic units for comparison, as LU $\mathrm{U}_{50}$ per 1 million NK-92 cells at 193 8-hours, there were dose-dependent losses of overall activity following irradiation that were amplified 194 when decreased cell survival was considered in combination with loss of function (Table 1). Activity 195 monitored by $\mathrm{LU}_{50}$ s one day after $10 \mathrm{~Gy}$ was consistently less than $30 \%$ of the non-irradiated NK-92 cells 196 in replicate experiments (data not shown).

Table 1. Compound Effects of Viable Cell Recovery and Impaired Lytic Activity on NK-92 Cell Functionality One Day after Irradiation* 


\begin{tabular}{|c|c|c|c|c|c|c|c|}
$\begin{array}{c}\text { Irradiation } \\
\text { (Grays) }\end{array}$ & $\begin{array}{c}\text { \% Control } \\
\text { viable cell } \\
\text { recovery }\end{array}$ & $\begin{array}{c}\text { Serial } \\
\text { KF }\end{array}$ & $\begin{array}{c}\text { \% Control } \\
\text { KF }\end{array}$ & $\begin{array}{c}\text { \% Remaining } \\
\text { functional activity } \\
\text { based on KF }\end{array}$ & $\begin{array}{c}\text { LU } \mathbf{U}_{\mathbf{5 0}} \text { per 10 } \\
\text { NK-92 cells }\end{array}$ & $\begin{array}{c}\text { \% Control } \\
\text { lytic units }\end{array}$ & $\begin{array}{c}\text { \% Remaining } \\
\text { functional } \\
\text { activity based on } \\
\text { lytic units }\end{array}$ \\
\hline 0 & 100 & 5.6 & 100 & 100 & 815.5 & 100 & 100 \\
2.5 & 53.4 & 3.9 & 69.6 & 37.2 & 419.8 & 51.5 & 27.5 \\
5 & 56.8 & 2.5 & 44.6 & 25.4 & 215.9 & 26.5 & 15.0 \\
10 & 39.0 & 1.5 & 26.8 & 10.4 & 23.8 & 2.9 & 1.1 \\
\hline
\end{tabular}

*Raji 'target' cells at E:T 1:16 after 8 hrs. This representative experiment was comparable to two other replicate experiments. + Cells proliferated to increase $75-80 \%$ from time of initial culture without irradiation.

\#Calculated as the fraction representing viable cell recovery multiplied by the fraction of control KF.

${ }^{\wedge}$ Calculated as the fraction representing viable cell recovery multiplied by the fraction of lytic unit activity that was retained compared to untreated NK-92 cells.

197 Fas (CD95) receptor ligation weakens NK-92 serial killing, especially one day after irradiation

198 Fas-ligand is expressed by many tumors and is a means by which these tumors can engage Fas-receptor 199 on NK- and/or T- cells and thereby initiate "suicide" of the effector lymphocytes (29) and protection of 200 the tumor cells. To evaluate if Fas ligation could also affect NK-92 cells, first, we monitored Fas/CD95 expression on NK-92 cells, then discovered a lack of NK-92 cell "suicide" responsiveness to Fas-ligation and, last, observed detrimental effects of Fas-ligation on serial killing. Jurkat cells that readily die after ligation of their membrane-bound Fas were used as positive controls for detection of Fas expression and induction of cellular "suicide" by anti-Fas antibodies (30). Jurkat and NK-92 cells were stained with AF647-anti-CD95/Fas and analyzed by flow cytometry. Figure 3A shows that $>95 \%$ of Jurkat and NK92 cells were positive for CD95/Fas. Furthermore, NK-92 cells expressed more Fas than Jurkat cells, with

207 a higher median fluorescence index (MFI) of 6,339 that remained high after irradiation, compared to an MFI of 2,263 for Jurkat cells. It should be noted that within a day, a subpopulation of $20 \%$ of the irradiated NK-92 cells had reduced levels of Fas (MFI 1851) (Figure 3A, with a 43\% subpopulation in a replicate experiment).

211 Following detection of CD95 on NK-92 cells, we then incubated non-irradiated and 10 Gy irradiated NK-

21292 cells with anti-Fas IgM overnight and subsequently examined these cells for apoptotic or necrotic

213 death. Cells were stained with FITC-annexin V, which binds to phosphatidylserine externalized on

214 apoptotic cells and also binds to the internal phosphatidyl serine of permeable necrotic cells. The Jurkat 
cells responded strongly to anti-Fas ligation, with 99\% annexin V positive (Figure 3B). In contrast, both non-irradiated and irradiated NK-92 cells responded weakly to Fas ligation, with a $<10 \%$ increase in

217 annexin V-positive cells compared to the control cells (Figure 3B). At least one third of all the annexin

218 V-positive cells also stained positive with 7-AAD, indicating that a substantial fraction of these cells had 219 progressed to necrosis (not illustrated). Despite NK-92 cell resistance to death following Fas ligation, 220 anti-Fas treatment did affect the irradiated, non-necrotic NK-92 cells, in form of shrunken cells (as 221 indicated by lower forward scatter (FSC)) (Figure 3C).

222 In contrast to the minimal effects on NK-viability, the effects on NK-92 cytotoxic functionality were 223 substantial after Fas-ligation. Non-irradiated or 10 Gy irradiated NK-92 cells were preincubated for one 224 day without antibody, with an IgM isotype control, or with anti-Fas IgM. The cytotoxic capacity of these 225 cells was then tested against K562 targets with the continuing presence of the antibodies in the ${ }^{51} \mathrm{Cr}$ 226 release assays. Because K562 cells lack Fas, they were used as tumor 'target' cells, which prevented 227 addition of anti-Fas-initiated "suicide" to the target cell killing by NK-92 cells. Figure 4A shows that 228 non-irradiated cells were able to maintain high cytotoxic activity following overnight Fas ligation, 229 reaching $80 \%$ killing compared to $80 \%$ - $83 \%$ killing by control and IgM treated cells respectively at 230 similar E:T ratios. By $\mathrm{LU}_{50}$, anti-Fas treated NK-92 cells retained $\sim 50 \%$ activity compared to the $\operatorname{IgM}$ 231 isotype control (insert, Figure 4A). Following 10 Gy irradiation, however, anti-Fas treated NK-92 232 cytotoxic activity was significantly decreased, reaching $44 \%$ killing compared to $74 \%$ - $77 \%$ killing by 233 control and IgM treated cells respectively (Figure 4B). The insert of Figure 4B illustrates that anti-Fas 234 treated, 10 Gy irradiated NK-92 cells retained just < 5\% activity by $\mathrm{LU}_{50}$ compared to control cells.

235 Figure 4C illustrates KF measurements of serial killing and emphasizes that this function is extremely 236 vulnerable to Fas-ligation after irradiation of the NK-92 cells. Regression analyses comparing the effects 237 of only Fas-ligation or only irradiation to the combination of both Fas-ligation and irradiation, revealed 238 synergistic rather than additive damage to cytotoxicity $(\mathrm{p}<0.001)$. 
241 While one would like to know if primary host NK cell attack of adoptively transferred NK-92 tumor cells

242 has effects on the cytotoxic functionality of NK-92 cells (even if the NK-92 cells were to resist attack),

243 the investigation is technically stymied because both the primary NK and NK-92 cells would be

244 concurrently mediating 'target cell' death. It would be impossible to sort out the contributions of each

245 cell. Instead, we simply determined if irradiated NK-92 cells could be attacked by the patients' own NK

246 cells, which would be another means by which efficacy of NK-92 cell adoptive therapy could be

247 compromised in vivo. The susceptibility of non-irradiated NK-92 cells to primary NK and IL-2 induced

248 LAK cell attack has been well-documented $(31,32)$. We wanted to assess if this susceptibility was

249 increased for irradiated NK-92 cells. This possibility is supported by the induction of stress ligands after

250 irradiation and these ligands can serve as recognition molecules for NK cells (33).

251 In preliminary experiments, we tested the ability of resting bNK or LAK cells to kill NK-92 cells. We

252 used K562 cells as control 'targets' to confirm that the healthy donors' bNK and LAK cells had good

253 cytotoxic activity, whether or not their NK cells killed NK-92 cells. The NK-92 target cells were either

254 non-irradiated or irradiated with $10 \mathrm{~Gy}$ and cultured overnight before assay. Irradiated NK-92 cells were

255 more sensitive to killing by bNK cells than non-irradiated NK-92 cells (Table 2), while the non-irradiated

256 NK-92 cells were killed to a lesser extent than K562 cells (as previously reported). LAK cells were able

257 to kill both non-irradiated and irradiated NK-92 cells as indicated for donor SC-6617, where a clear

258 increase in LAK $v s$. bNK anti-NK-92 cell killing was observed (Table 2). These limited experiments

259 indicate that after irradiation, NK-92 cells retain and/or increase ligands for NK attack and thus are

260 susceptible to innate immune elimination in vivo, indicating a third consideration that could be addressed

261 by avoiding irradiation and/or by genetic engineering.

\begin{tabular}{|c|c|c|c|c|c|}
\hline \multicolumn{5}{|c|}{ Table 2. Primary Blood NK and LAK Killing of NK-92 Cells at 4 Hours } \\
\hline \multirow{2}{*}{$\begin{array}{c}\text { NK Activation } \\
\text { State }\end{array}$} & \multirow{2}{*}{ Donor \# } & $\begin{array}{c}\text { E:T } \\
\text { (PBMC:NK-92) }\end{array}$ & Non-irradiated NK-92 & 10 Gy irradiated NK-92 $_{1}$ K562 \\
\hline
\end{tabular}




\begin{tabular}{|c|c|c|c|c|c|}
\hline \multirow{4}{*}{ Unstimulated } & SC-0394 & 107:1 & $7 \% *$ & $15 \%$ & $76 \%$ \\
\hline & SC-3663 & $33.5: 1$ & $2 \% *$ & $6 \% *$ & $23 \%$ \\
\hline & SC-6617 & $60: 1$ & $8 \% *$ & $19 \%$ & $13 \%$ \\
\hline & SC-0975 & $48: 1$ & $7 \% *$ & $12 \%$ & $11 \%$ \\
\hline \multirow{4}{*}{ IL-2 LAK } & SC-0394 & $23: 01$ & $8 \% *$ & ND & $96 \%$ \\
\hline & SC-3663 & $15.2: 1$ & $1 \% *$ & $4 \% *$ & $47 \%$ \\
\hline & SC-6617 & $55: 1$ & $17 \%$ & $31 \%$ & $78 \%$ \\
\hline & SC-0975 & $42: 1$ & $27 \%$ & $43 \%$ & $82 \%$ \\
\hline
\end{tabular}

\section{Discussion}

263 In this study we demonstrated the remarkable serial killing potential of NK-92 cells towards several

264 'target' cell types. We shed light on potential limitations to NK-92 cell-mediated serial killing and

265 therapeutic efficacy, specifically following irradiation, a current clinical practice preceding adoptive

266 transfer. We also tested NK-92 cell functionality remaining after Fas-ligation and documented NK-92

267 susceptibility to attack by circulating bNK cells, which are two potential limitations of adoptive cell

268 therapy. These findings suggest that NK-92 cells have immense potential in adoptive cancer

269 immunotherapy but should be carefully optimized before infusion into patients to ensure greatest

270 therapeutic efficacy.

271 Serial killing by NK-92 cells has been documented before; however, we directed our attention to potential

272 in vivo effects on serial killing. A previous study focused on the development of a droplet-based

273 cytotoxicity assay that utilized a lowest E:T of 1:3 and showed that $\sim 50 \%$ of observed NK-92 cells are

274 able to serially kill two or more K562 targets in 12 hours (34). Serial cytotoxicity has also been observed

275 with time-lapse cinematography using genetically modified, IL-2 producing NK92-MI cells. This study

276 indicated that one NK-92 cell could kill as many as 14 target HeLa cells over six hours (27). Many

277 studies have used standard radioactive release assays to characterize NK-92 killing towards various cell

278 types, but, without an excess of 'targets', these assays were unable to address NK-92 serial cytotoxicity.

279 Our results confirm NK-92 serial killers and show that serial killing is target cell type dependent, with 
Raji and Daudi targets reaching KFs > 10, while K562 targets are far less susceptible to serial killing with

281 KFs $<3.0$ (Figure 1).

282 This variation in killing for different 'target' cells, as determined by KFs, may be due to differences in

283 ligands on target cells that engage the diverse activation receptors of NK-92 cells. One possible

284 explanation for low killing towards K562 cells is that NK-92 cells poorly express the receptor NKG2D, in 285 contrast to high NKG2D-expressing KHYG-1 cell line that kills K562 cells much more effectively (35).

286 Furthermore, K562s produce the granzyme B inhibitor PI-9, making them less susceptible to killing via

287 granzyme B, which is predominately used by NK-92 cells but overcome by granzyme M used by KHGY-

2881 (36). It is possible that cleavage of NKG2D ligands by metalloproteinases such as ADAM10 (37)

289 combined with NKG2D downregulation during cytotoxic activation (38) affects NK-92 killing of K562s.

290 This loss of NKG2D may also explain the plateau in killing that we observed towards K562 after 6 hours, 291 and which has been observed for up to 12 hours elsewhere (34). A limitation of the KF method (5) to 292 detect serial killing is that it measures the simple average number of targets killed per effector, rather than 293 identifying the fraction of effectors engaged in killing within the NK cell population. Regardless, the KF 294 method is still optimal (in terms of statistical validity, time, labor, and cost savings) to screen treatments used prior to adoptive cell transfer for their effects on serial killing.

296 Prior studies have investigated the effects of irradiation on NK-92 cell-mediated killing; however, these 297 studies used shorter cytotoxicity assays with different target cells and reported that NK-92 cytotoxic 298 capacity is mostly retained for at least 24 hours following irradiation $(22,39)$. Another study, using haNK 299 cells 24-hours post irradiation saw an increase in cytotoxicity toward multiple carcinoma cell lines 300 compared to haNK cell killing immediately post irradiation (40). These findings are in contrast to our 301 findings with unmodified NK-92 cells, which show decreases in killing toward Raji cells one day after 302 irradiation (Figure 2) as well as K562 cells (unpublished). Possible explanations for the differences 303 between these findings include haNK cell endogenous expression of IL-2, while our cells were 304 supplemented with $1000 \mathrm{U} / \mathrm{ml} \mathrm{IL-2,} \mathrm{lymphoid} \mathrm{versus} \mathrm{carcinoma} \mathrm{target} \mathrm{cells,} \mathrm{as} \mathrm{well} \mathrm{as} \mathrm{the} \mathrm{18-hr} \mathrm{release}$ 
assays used for the haNK cells, which may allow for detection of longer-term killing potential than our 8hour release assays. In this report, we extended the effects of irradiation to effects on cytotoxicity after

307 Fas-ligation and to NK-92 cellular susceptibility to potential attack by patient NK cells. First, we 308 observed a decrease in NK-92 cell viability and viable cell recovery (Table 1), as well as a consistent 309 decrease in serial cytotoxicity one day after irradiation, even at lower doses of 2.5 - 5 Gy (Figure 2).

310 Notably, this decrease in killing was absent when NK-92 cells were assayed immediately following up to 31120 Gy irradiation. This initial retention of activity indicates that therapeutic cell lines should be used 312 immediately post irradiation to maximize cytotoxicity in vivo, as in the design of one phase I trial (21). 313 Ideally, an alternative anti-proliferative approach for cell lines used in adoptive therapies would be used.

314 The profound effects of irradiation on NK-92 cytotoxic capacity indicate that radiation effects extend beyond DNA damage and likely include direct damage to proteins $(41,42)$. Ionizing radiation produces radiolysis products, such as reactive oxygen species that inactivate proteome functions including those involved in killing and DNA repair (39). Low energy electron irradiation, as an alternative to gamma irradiation, inhibits NK-92 cell proliferation while maintaining higher cytotoxic capacity and for longer periods of time and could therefore be considered for clinical applications (39). This report also indicates that 2-hours after 10 Gy gamma irradiation, there is lower expression of genes encoding multiple

321 pathways that are critical to cell-mediated cytotoxicity (39). Considering the additional impact of direct

322 proteome damage by irradiation, alternative treatments such as induction of genetically introduced type II 323 restriction enzymes and pretreatment of cells with certain topoisomerase inhibitors (Hudig et. al., 324 unpublished results) that only inflict damage to DNA could be used prior to adoptive transfer (42).

325 We discovered an Achille's heel for irradiated NK-92 cells, Fas/CD95, which has previously been noted 326 on the majority of activated NK cells $(43,44)$ and on NK-92 cells $(45)$. Despite high expression of CD95, 327 anti-Fas antibodies alone failed to affect proliferation or to initiate death of non-irradiated NK-92 cells 328 within one day, even though the non-irradiated cells did respond to Fas-ligation by shrinking in size. One 329 possible explanation for the NK-92 cell's low sensitivity to death after anti-Fas ligation is that there are 
two pathways of Fas-mediated death, one of which relies on mitochondrial signal amplification. This type

331 II, intrinsic pathway is slow and readily inhibited by expression of the Bcl-2 family of apoptotic proteins

332 (29,46). Another possible explanation is that NK-92 cells may express wild-type PI-9, which inhibits the

333 caspase-dependent Fas/FasL-mediated death pathways (47). Intrinsic resistance to Fas-ligation is also

334 indicated by evidence that NK-92 cells constitutively produce soluble Fas ligand (39).

335 Even though the NK-92 cells resisted death by Fas, they did respond with decreased cytotoxic activity.

336 Fas-ligation alone could decrease NK-92 cytotoxicity to Raji cells, but these effects were always two-fold

337 or less for non-irradiated effector cells. However, for irradiated NK-92 cells the anti-Fas effect was

338 remarkably stronger, with just $10 \%$ or less of control killing remaining. In synergy, Fas-ligation and

339 irradiation profoundly reduced cytotoxicity (Figure 4). One possible explanation for this synergistic

340 effect, seen with cytotoxicity but not with viability, is that the cell shrinkage that occurred with Fas-

341 ligated irradiated, non-necrotic NK-92 cells impaired their activity. This shrinkage, that was absent from

342 Fas-ligated non-irradiated cells, is related to dehydration and has been reported as an early indicator of

343 cell death (48).

344 These findings suggest a serious risk for engagement of CD95 as a mechanism to hamper NK-92 cell

345 therapeutic efficacy in vivo, especially if a patient's tumor cells express the counter Fas-ligand (Fas-

346 L/CD178). After irradiation, NK-92 cells appear to have normal viability in the face of Fas-mediated

347 death receptor ligation but are considerably less-effective killers. A logical next step could be to remove

348 CD95 from NK-92 cells in order to reduce their susceptibility to rapid death via the Fas pathway. Recent

349 advances in CRISPR/Cas-9 have made the methodology a more efficient way to genetically engineer NK-

35092 cells, including the implementation of multiple genetic changes at one time (49).

351 Having discovered that irradiation affects NK-92 cell susceptibility to Fas-ligation, we queried if

352 irradiation would also make NK-92 cells more vulnerable to attack when encountered by patient NK cells.

353 We found that irradiated NK-92 cells are susceptible to attack by both unstimulated and IL-2 LAK bNK, 
whereas non-irradiated NK-92 cells were more resistant to killing (Table 2). These results contrast with previous reports in which substantial killing to non-irradiated NK-92 cells (comparable to K562) was observed $(31,32)$. A technical consideration may contribute to these differences: the IL-2 concentration

357 used to maintain the susceptible NK-92 cells was $20 \mathrm{U} / \mathrm{ml}$, while we used $1000 \mathrm{U} / \mathrm{ml} \mathrm{IL}-2$. Our results are 358 preliminary due to a limited number of NK cell donors but do indicate that, after irradiation, NK-92 cells 359 may become more sensitive to attack by circulating NK cells. This NK -mediated attack could potentially 360 be further promoted by antibody-dependent cell-mediated cytotoxicity (ADCC) supported by IgG 361 antibodies that patients develop to NK-92 cell MHC class I proteins (21). We suggest that increased 362 sensitivity to host cell attack be monitored whenever NK-92 cells are genetically modified or are treated 363 before adoptive transfer.

364 Our research was limited to the cytotoxic NK line NK-92, which is only one of several lines that are being 365 evaluated for immunotherapies (Additional File 1: Table S1). Our study is also limited in that all assays 366 were conducted in vitro. Nonetheless, we were able to underscore the importance of serial killing as a 367 critical variable that may be compromised by pretreatments such as irradiation and by in vivo conditions 368 such as intratumor Fas ligand and bNK attack. The research indicates that other cells lines should be 369 similarly evaluated for potential on serial killing. Tumor counter-ligands other than Fas that stimulate NK 370 inhibitory receptors may also profoundly compromise serial killing, a possibility that is yet to be

371 explored. A broad implication is that it may become clinically worthwhile to genetically profile ligands 372 of a tumor environment that affect NK serial killing to select the best NK cell line for immunotherapy.

\section{Conclusions}

374 Based on these findings, our data warrant urgent changes for clinical immunotherapy. We indicated that 375 non-irradiated NK-92 cells are more effective than irradiated NK-92 cells in three ways: increased serial 376 killing, resistance to Fas-ligation effects, and resistance to attack by NK or LAK cells. Preclinical studies 377 of therapeutic efficacy testing non-irradiated NK-92 cells in murine models are overlooking these three 378 key limitations and thus are probably overestimating predicted outcomes for patients. Of greatest 
379 importance to improved clinical outcomes is the need to replace irradiation with alternative methods of 380 anti-proliferative pretreatment and to test any alternative method for effects on serial killing of tumor 381 cells.

\section{$382 \quad$ Figures}

Figure 1. NK-92 cell cytotoxicity and serial killing frequencies of Raji, Daudi and K562 targets. Each colored line/symbol represents \%specific release at a different E:T. Dashed lines represent the expected \% dead targets if one target was killed by one effector at an E:T. A. Raji cells as 'targets'. B. Daudi cells as 'targets'. C. K562 cells as 'targets'. The three targets were assayed concurrently. The standard deviations for each data point were less than $2 \%$ specific ${ }^{51} \mathrm{Cr}$ release. The cytotoxicity towards Raji cells is significantly greater than Daudi and K562 cells (E:T 1:32 linear regression p $<0.01 \& p$ $<0.001$, respectively).

Figure 2. Effects of irradiation on serial killing. NK-92 cells were irradiated on the day of experiment (A) or the day before the experiment and cultured with IL-2 (B). Cytotoxicity was measured after 8 hours. The KFs for the 1:16 E:Ts are indicated in the two boxes in the middle of the figure. E:Ts are graphed on a $\log _{10}$ scale. [***p-value $<0.001$ via regression analysis]

Figure 3. Expression of CD95 (Fas) by NK-92 cells and effects of Fas-ligation on their viability. Fas-sensitive Jurkat cells were used as positive controls for Fas expression and death after a day of Fas ligation. Dead cells are indicated by binding of FITC-labeled annexin V to phosphatidyl serine that is externalized in the plasma membranes of dying cells. A. Surface expression of Fas/CD95. 1. Expression of Fas by Jurkat cells. 1a. Unlabeled cells. 1b. Cells labeled with mAb anti-CD95 Fas. 2. Expression of Fas by non-irradiated NK-92 cells. $2 a \& 2 b$ are as indicated for $1 \mathrm{a} \& 1 \mathrm{~b}$. 3. Expression of Fas by NK-92 cells irradiated with 10 Gy. 3a\&3b are as indicated for $1 \mathrm{a} \& 1 \mathrm{~b}$. B. Induction of death with control IgM or IgM anti-Fas in overnight culture. By Overton subtraction, the conversion from annexin V-low to annexin V-high cells (apoptotic and necrotic) was 52\% for Jurkat cells, 5.8\% for non- 
irradiated NK-92 cells, and 9.2\% for the irradiated NK-92 cells $(* p<0.05)$. C. Changes in cellular size

404 (forward scatter) in response to irradiation and Fas-ligation. 1. Non-irradiated cells cultured with

405 media, IgM isotype, or anti-Fas IgM. 2. Cells one day after 10 Gy irradiation, cultured with media, IgM

406 isotype, or anti-Fas IgM.

Figure 4. Effects of CD95 (Fas)-ligation on serial killing by non-irradiated and irradiated NK-92

cells. Non-irradiated or 10Gy irradiated cells were cultured for one day with nothing, $1 \mathrm{ug} / \mathrm{ml}$ control IgM or IgM anti-Fas. Because K562 cells lack Fas, they were used as tumor 'target' cells to prevent addition of anti-Fas-initiated "suicide" to target cell killing by NK-92 cells. Cytotoxic activity towards K562 cells was measured after 8 hours. E:T ratios are approximate. A. Anti-Fas ligation limited to non-irradiated NK-92 cells. Insert: $\mathrm{LU}_{50} / 1 \mathrm{M}$ non-irradiated NK-92 cell, by treatment. B. Anti-Fas ligation of 10 Gy irradiated NK-92 cells. Insert: LU50/1M irradiated NK-92 cell, by treatment. [***p-value $<0.001$ via regression analysis]

Figure 5. Killing of NK-92 cells by either resting or activated primary blood NK cells. The susceptibility of NK-92 tumor cells to attack by primary peripheral blood NK cells from healthy donors was investigated using NK-92 cells that were either non-irradiated or were irradiated with 10 Gy and then cultured for one day. The primary NK cells were either resting or activated by three days culture with $1000 \mathrm{u} / \mathrm{ml} \mathrm{IL-2.} \mathrm{Cytotoxicity} \mathrm{was} \mathrm{measured} \mathrm{using} \mathrm{4-hour}{ }^{51} \mathrm{Cr}$-release assays; the gray dashed line reflects the $10 \%$ threshold for positive killing. The results illustrated represent concurrent assays made with E:Ts of viable effector NK cells from a single donor SC-0975 and are representative of 4 experiments each with a different donor. A. Killing of NK-92 cells by resting primary NK cells. $\mathrm{LU}_{50} \mathrm{~S}$ were excluded for unstimulated NK cells because killing was over $10 \%$ for only the highest E:T. B.

424 Killing of NK-92 cells by activated primary NK cells. For both bNKs and LAKs, the cytotoxicity 425 towards non-irradiated versus irradiated NK-92 cells was significantly different at the highest E:T

426 (student t-test, * $\mathrm{p}<0.03, * * \mathrm{p}<0.005$ ). Insert for B. LU50/1M LAK bNK calculated using 427 TruCOUNT ${ }^{\mathrm{TM}}$ beads (Gating - Additional File 1: Figure S1). 


\section{Declarations}

429 Ethics approval and consent to participate The blood lymphocytes were collected by STEMCELL

430 Technologies (Vancouver, Canada) under their authorization to obtain blood from healthy donors.

431 Consent for publication Not applicable.

432 Availability of data and materials The data that support the findings of this study are included in this

433 published article and its supplementary information files. Additional data are available from the

434 corresponding author on reasonable request.

435 Competing interests Not applicable.

436 Funding This work was supported in part by grants from the Nevada IDeA Network of Biomedical

437 Research Excellence (NIH GM103440), a Nevada Undergraduate Research Award, a UNR Foundation

438 Award and an NIH P30 for the Cytometry Center (NIH GM110767).

439 Authors' contributions LNG, DH, and MG designed the study with helpful suggestions from VL and 440 RM. LNG, DH, MG, and JCA performed the experiments and acquired data. LNG, DH, MG, and JSG

441 analyzed the data. LNG and DH wrote the manuscript. All authors were involved in the critical review

442 and editing of the manuscript and approved the final version.

443 Acknowledgements The authors thank William J. Murphy, Ph.D. for constructive suggestions, Carl 444 Ware, Ph.D. for helpful suggestions concerning measurement of anti-Fas effects, Myung Chul Jo for 445 irradiating the cells at UNR, Dana E. Reed for mycoplasma testing of cell lines, David M. White for 446 assistance with flow cytometry, and Matthew Isom for technical support.

\section{Additional Files}



CD16.

Additional file 2.pdf, Figure S1. Flow cytometry to determine the number of primary NK cells within peripheral blood mononuclear cells. A. Gating sequence. 1. First, beads were gated by high

453 Pacific Orange signal. 2. Cells without debris were gated by forward scatter (FSC-A) v side scatter (SSC-

454 A). 3. Single cells (without doublets) were gated by SSC height versus SSC width. 4. All cells were gated 455 on by Pacific Blue anti-CD45. 5. Live cells were gated by Boolean not gate, taking 7-AAD positive cells 456 as dead cells. 6. T-cells were gated out by Boolean not gate of FITC anti-CD3 positive T-cells. 7. NK 457 cells were gated on as AF647 anti-CD244 positive cells. CD244 is expressed by CD8+ T-cells (gated out with CD3) and all NK cells (24). 8. NK cell staining for BV711 anti-CD16/CD56. B. Expression of CD16A \& CD56 by resting and IL-2 activated (LAK) bNK cells. Light blue samples are the unstained control cells respective for each group. Red samples are stained CD16A and/or CD56. 1. Unstimulated bNK cells. 2. Interleukin-2 activated LAK bNK cells.

Additional file 3.pdf, Figure S2. Killing of NK-92 cells by either resting or activated primary blood NK cells. The susceptibility of NK-92 cells to attack by primary peripheral blood NK cells from healthy donors was investigated using NK-92 cells that were either non-irradiated or were irradiated with 10 Gy and then cultured for one day. The primary NK cells were either resting or activated by three days culture with $1000 \mathrm{u} / \mathrm{ml} \mathrm{IL-2.} \mathrm{Cytotoxicity} \mathrm{was} \mathrm{measured} \mathrm{using} \mathrm{4-hour}{ }^{51} \mathrm{Cr}$-release assays; the horizontal gray

467 dashed line reflects the $10 \%$ threshold for positive killing. The results illustrated represent assays of 468 multiple donors across multiple experiments.

\section{References}

470 1. Miller JS, Lanier LL. Natural killer cells in cancer immunotherapy. Annual Review of Cancer $471 \quad$ Biology. 2019;3(1). 
2. Hodgins JJ, Khan ST, Park MM, Auer RC, Ardolino M. Killers 2.0: NK cell therapies at the forefront of cancer control. Journal of Clinical Investigation. 2019;129(9):3499-510.

3. Heipertz EL, Zynda ER, Stav-Noraas TE, Hungler AD, Boucher SE, Kaur N, et al. Current Perspectives on "Off-The-Shelf" Allogeneic NK and CAR-NK Cell Therapies. Frontiers in Immunology. 2021 Dec 1;12.

4. Isaaz S, Baetz K, Olsen K, Podack E, Griffiths GM. Serial killing by cytotoxic T lymphocytes: T cell receptor triggers degranulation, re-filling of the lytic granules and secretion of lytic proteins via a non-granule pathway. European Journal of Immunology. 1995 Apr;25(4).

5. Bhat R, Watzl C. Serial Killing of Tumor Cells by Human Natural Killer Cells - Enhancement by Therapeutic Antibodies. PLoS ONE. 2007 Mar 28;2(3):e326.

6. Vanherberghen B, Olofsson PE, Forslund E, Sternberg-Simon M, Khorshidi MA, Pacouret S, et al. Classification of human natural killer cells based on migration behavior and cytotoxic response. Blood. 2013 Feb 21;121(8).

7. Perussia B, Trinchieri G. Inactivation of natural killer cell cytotoxic activity after interaction with target cells. Journal of Immunobiology. 1981 Feb;126(2):754-8.

8. Romain G, Senyukov V, Rey-Villamizar N, Merouane A, Kelton W, Liadi I, et al. Antibody Fc engineering improves frequency and promotes kinetic boosting of serial killing mediated by NK cells. Blood. 2014 Nov 20;124(22).

9. Srpan K, Ambrose A, Karampatzakis A, Saeed M, Cartwright ANR, Guldevall K, et al. Shedding of CD16 disassembles the NK cell immune synapse and boosts serial engagement of target cells. Journal of Cell Biology. 2018;217(9).

10. Klingemann H, Boissel L, Toneguzzo F. Natural killer cells for immunotherapy - Advantages of the NK-92 cell line over blood NK cells. Frontiers in Immunology. 2016 Mar 14;7:91.

11. Vivier E, Raulet DH, Moretta A, Caligiuri MA, Zitvogel L, Lanier LL, et al. Innate or Adaptive Immunity? The Example of Natural Killer Cells. Science. 2011 Jan 7;331(6013).

12. Gunesch JT, Angelo LS, Mahapatra S, Deering RP, Kowalko JE, Sleiman P, et al. Genome-wide analyses and functional profiling of human NK cell lines. Molecular Immunology. 2019 Nov $1 ; 115: 64-75$.

13. Yang HG, Kang MC, Kim TY, Hwang I, Jin HT, Sung YC, et al. Discovery of a novel natural killer cell line with distinct immunostimulatory and proliferative potential as an alternative platform for cancer immunotherapy. Journal for ImmunoTherapy of Cancer. 2019 May 24;7(1).

14. Solocinski K, Padget MR, Fabian KP, Wolfson B, Cecchi F, Hembrough T, et al. Overcoming hypoxia-induced functional suppression of NK cells. Journal for ImmunoTherapy of Cancer. 2020;8(1).

15. Suck G, Odendahl M, Nowakowska P, Seidl C, Wels WS, Klingemann HG, et al. NK-92: an 'offthe-shelf therapeutic' for adoptive natural killer cell-based cancer immunotherapy. Cancer Immunology, Immunotherapy. 2016 Apr 1;65(4):485-92. 
16. Jochems C, Hodge JW, Fantini M, Tsang KY, Vandeveer AJ, Gulley JL, et al. ADCC employing an NK cell line (haNK) expressing the high affinity CD16 allele with avelumab, an anti-PD-L1 antibody. International Journal of Cancer. 2017 Aug 1;141(3):583-93.

17. Fabian KP, Padget MR, Donahue RN, Solocinski K, Robbins Y, Allen CT, et al. PD-L1 targeting high-affinity NK (t-haNK) cells induce direct antitumor effects and target suppressive MDSC populations. Journal for ImmunoTherapy of Cancer. 2020 May 20;8(1).

18. Arai S, Meagher R, Swearingen M, Myint H, Rich E, Martinson J, et al. Infusion of the allogeneic cell line NK-92 in patients with advanced renal cell cancer or melanoma: A phase I trial. Cytotherapy. 2008;10(6).

19. Tonn T, Schwabe D, Klingemann HG, Becker S, Esser R, Koehl U, et al. Treatment of patients with advanced cancer with the natural killer cell line NK-92. Cytotherapy. 2013 Dec;15(12).

20. Burger M. Intracranial Injection of NK-92/5.28.z Cells in Patients With Recurrent HER2-positive Glioblastoma (CAR2BRAIN). Identifier NCT03383978. National Library of Medicine U.S.; 2017.

21. Williams BA, Law AD, Routy B, denHollander N, Gupta V, Wang XH, et al. A phase I trial of NK-92 cells for refractory hematological malignancies relapsing after autologous hematopoietic cell transplantation shows safety and evidence of efficacy. Oncotarget. 2017;8(51).

22. Klingemann H-G, Wong E, Maki G. A cytotoxic NK-cell line (NK-92) for ex vivo purging of leukemia from blood. Biology of Blood and Marrow Transplantation. 1996 May;2(2):68-75.

23. Golstein P. Cytotoxicity, Mechanisms of. Encyclopedia of Immunology. 1998 Jan 1;732-4.

24. McNerney ME, Lee KM, Kumar V. 2B4 (CD244) is a non-MHC binding receptor with multiple functions on natural killer cells and CD8+ T cells. Molecular Immunology. 2005;42(4 SPEC. ISS.).

25. Overton WR. Modified histogram subtraction technique for analysis of flow cytometry data. Cytometry [Internet]. 1988 [cited 2021 Nov 18];9(6):619-26. Available from: https://pubmed.ncbi.nlm.nih.gov/3061754/

26. Alter G, Malenfant JM, Altfeld M. CD107a as a functional marker for the identification of natural killer cell activity. Journal of Immunological Methods. 2004;294(1-2).

27. Choi PJ, Mitchison TJ. Imaging burst kinetics and spatial coordination during serial killing by single natural killer cells. Proceedings of the National Academy of Sciences of the United States of America. 2013;110(16).

28. Xu Y, Zhou S, Lam YW, Pang SW. Dynamics of natural Killer cells cytotoxicity in Microwell arrays with connecting channels. Frontiers in Immunology. 2017;8:998.

29. Timmer T, de Vries EGE, de Jong S. Fas receptor-mediated apoptosis: A clinical application? Journal of Pathology. 2002;196(2):125-34.

30. Matsui H, Tsuji S, Nishimura H, Nagasawa S. Activation of the alternative pathway of complement by apoptotic Jurkat cells. FEBS Letters. 1994;351(3). 
31. Bergman H, Sissala N, Hägerstrand H, Lindqvist C. Human NK-92 cells function as target cells for human NK Cells - Implications for CAR NK-92 therapies. Anticancer Research. 2020;40(10):5355-9.

32. Bergman H, Lindqvist C. Human IL-15 inhibits NK cells specific for human NK-92 cells. Anticancer Research. 2021 Jul 1;41(7):3281-5.

33. Ames E, Canter RJ, Grossenbacher SK, Mac S, Smith RC, Monjazeb AM, et al. Enhanced targeting of stem-like solid tumor cells with radiation and natural killer cells. OncoImmunology. 2015;4(9).

34. Antona S, Platzman I, Spatz JP. Droplet-Based Cytotoxicity Assay: Implementation of TimeEfficient Screening of Antitumor Activity of Natural Killer Cells. ACS Omega. 2020;5(38).

35. Suck G, Branch DR, Smyth MJ, Miller RG, Vergidis J, Fahim S, et al. KHYG-1, a model for the study of enhanced natural killer cell cytotoxicity. Experimental Hematology. 2005;33(10).

36. Classen CF, Ushmorov A, Bird P, Debatin KM. The granzyme B inhibitor PI-9 is differentially expressed in all main subtypes of pediatric acute lymphoblastic leukemias. Haematologica. 2004;89(11).

37. Zingoni A, Vulpis E, Loconte L, Santoni A. NKG2D Ligand Shedding in Response to Stress: Role of ADAM10. Frontiers in Immunology. 2020 Mar 25;11.

38. Molfetta R, Quatrini L, Santoni A, Paolini R. Regulation of NKG2D-dependent NK Cell functions: The Yin and the Yang of receptor endocytosis. International Journal of Molecular Sciences. 2017;18(8).

39. Walcher L, Kistenmacher AK, Sommer C, Böhlen S, Ziemann C, Dehmel S, et al. Low Energy Electron Irradiation Is a Potent Alternative to Gamma Irradiation for the Inactivation of (CAR)NK-92 Cells in ATMP Manufacturing. Frontiers in Immunology. 2021 Jun 4;12.

40. Jochems C, Hodge JW, Fantini M, Fujii R, Maurice Morillon YI, Greiner JW, et al. An NK cell line (haNK) expressing high levels of granzyme and engineered to express the high affinity CD16 allele. Oncotarget [Internet]. 2016 Dec;7(52):86359-73. Available from: www.impactjournals.com/oncotarget/

41. Krisko A, Radman M. Protein damage and death by radiation in Escherichia coli and Deinococcus radiodurans. Proceedings of the National Academy of Sciences of the United States of America. 2010;107(32).

42. Radman M. Protein damage, radiation sensitivity and aging. DNA Repair. 2016 Aug 1;44:186-92.

43. Robertson MJ, Manley TJ, Pichert G, Cameron C, Cochran KJ, Levine H, et al. Functional consequences of APO-1/fas (CD95) antigen expression by normal and neoplastic hematopoietic cells. Leukemia and Lymphoma. 1995;17(1-2).

44. Medvedev AE, Johnsen AC, Haux J, Steinkjer B, Egeberg K, Lynch DH, et al. Regulation of Fas and Fas-ligand expression in NK cells by cytokines and the involvement of FAS-ligand in NK/LAK cell-mediated cytotoxicity. Cytokine. 1997;9(6).

45. Han R, Wu WQ, Wu XP, Liu CY. Effect of total flavonoids from the seeds of Astragali complanati on natural killer cell function. Journal of Ethnopharmacology. 2015;173. 
584 46. Barnhart BC, Alappat EC, Peter ME. The CD95 Type I/Type II model. Seminars in Immunology. $585 \quad 2003 ; 15(3): 185-93$.

586 47. Cunningham TD, Jiang X, Shapiro DJ. Expression of high levels of human proteinase inhibitor 9 587 blocks both perforin/granzyme and Fas/Fas ligand-mediated cytotoxicity. Cellular Immunology. $588 \quad 2007 ; 245(1)$.

589 48. Wlodkowic D, Skommer J, Darzynkiewicz Z. Cytometry of apoptosis. Historical perspective and 590 new advances. Experimental Oncology. 2012;34(3).

591 49. Huang RS, Shih HA, Lai MC, Chang YJ, Lin S. Enhanced NK-92 Cytotoxicity by CRISPR Genome Engineering Using Cas9 Ribonucleoproteins. Frontiers in Immunology. 2020;11. 


\section{Figures}

\section{Figure 1}

NK-92 cell cytotoxicity and serial killing frequencies of Raji, Daudi and K562 targets. Each colored line/symbol represents \%specific release at a different E:T. Dashed lines represent the expected \% dead targets if one target was killed by one effector at an E:T. A. Raji cells as 'targets'. B. Daudi cells as 'targets'. C. K562 cells as 'targets'. The three targets were assayed concurrently. The standard deviations for each data point were less than $2 \%$ specific $51 \mathrm{Cr}$ release. The cytotoxicity towards Raji cells is significantly greater than Daudi and K562 cells (E:T 1:32 linear regression $p<0.01 \& p<0.001$, respectively).

\section{Figure 2}

Effects of irradiation on serial killing. NK-92 cells were irradiated on the day of experiment (A) or the day before the experiment and cultured with IL-2 (B). Cytotoxicity was measured after 8 hours. The KFs for the 1:16 E:Ts are indicated in the two boxes in the middle of the figure. E:Ts are graphed on a log10 scale. $\left[{ }^{\star \star \star *} \mathrm{p}\right.$-value $<0.001$ via regression analysis]

\section{Figure 3}

Expression of CD95 (Fas) by NK-92 cells and effects of Fas-ligation on their viability. Fas-sensitive Jurkat cells were used as positive controls for Fas expression and death after a day of Fas ligation. Dead cells are indicated by binding of FITC-labeled annexin $V$ to phosphatidyl serine that is externalized in the plasma membranes of dying cells. A. Surface expression of Fas/CD95. 1. Expression of Fas by Jurkat cells. 1a. Unlabeled cells. 1b. Cells labeled with mAb anti-CD95 Fas. 2. Expression of Fas by nonirradiated NK-92 cells. 2a\&2b are as indicated for 1a\&1b. 3. Expression of Fas by NK-92 cells irradiated with $10 \mathrm{~Gy}$. 3a\&3b are as indicated for 1a\&1b. B. Induction of death with control IgM or IgM anti-Fas in overnight culture. By Overton subtraction, the conversion from annexin V-low to annexin V-high cells (apoptotic and necrotic) was 52\% for Jurkat cells, $5.8 \%$ for non- 18 irradiated NK-92 cells, and $9.2 \%$ for the irradiated NK-92 cells ( $\left.{ }^{*} p<0.05\right)$. C. Changes in cellular size (forward scatter) in response to irradiation and Fas-ligation. 1. Non-irradiated cells cultured with media, IgM isotype, or anti-Fas IgM. 2. Cells one day after $10 \mathrm{~Gy}$ irradiation, cultured with media, IgM isotype, or anti-Fas IgM. 
Effects of CD95 (Fas)-ligation on serial killing by non-irradiated and irradiated NK-92 cells. Non-irradiated or $10 \mathrm{~Gy}$ irradiated cells were cultured for one day with nothing, $1 \mathrm{ug} / \mathrm{ml}$ control $\mathrm{lgM}$ or $\operatorname{lgM}$ anti-Fas. Because K562 cells lack Fas, they were used as tumor 'target' cells to prevent addition of anti-Fasinitiated "suicide" to target cell killing by NK-92 cells. Cytotoxic activity towards K562 cells was measured after 8 hours. E:T ratios are approximate. A. Anti-Fas ligation limited to non-irradiated NK-92 cells. Insert: LU50/1M non-irradiated NK-92 cell, by treatment. B. Anti-Fas ligation of 10 Gy irradiated NK-92 cells. Insert: LU50/1M irradiated NK-92 cell, by treatment. [ ${ }^{\star \star \star} \mathrm{p}$-value $<0.001$ via regression analysis]

\section{Figure 5}

Killing of NK-92 cells by either resting or activated primary blood NK cells. The susceptibility of NK-92 tumor cells to attack by primary peripheral blood NK cells from healthy donors was investigated using NK-92 cells that were either non-irradiated or were irradiated with $10 \mathrm{~Gy}$ and then cultured for one day. The primary NK cells were either resting or activated by three days culture with $1000 \mathrm{u} / \mathrm{ml}$ IL-2. Cytotoxicity was measured using 4-hour 51 Cr-release assays; the gray dashed line reflects the $10 \%$ threshold for positive killing. The results illustrated represent concurrent assays made with E:Ts of viable effector NK cells from a single donor SC-0975 and are representative of 4 experiments each with a different donor. A. Killing of NK-92 cells by resting primary NK cells. LU50s were excluded for unstimulated NK cells because killing was over $10 \%$ for only the highest E:T. B. Killing of NK-92 cells by activated primary NK cells. For both bNKs and LAKs, the cytotoxicity towards non-irradiated versus irradiated NK-92 cells was significantly different at the highest E:T (student t-test, ${ }^{*} p<0.03,{ }^{*} p<0.005$ ). Insert for B. LU50/1M LAK bNK calculated using TruCOUNT TM ${ }^{\mathrm{TM}}$ beads (Gating - Additional File 1: Figure S1).

\section{Supplementary Files}

This is a list of supplementary files associated with this preprint. Click to download.

- Additionalfile1.pdf

- Additionalfile2.pdf

- AdditionalFile3.pdf 